\title{
LI-RADS in the year 2020 - Are you already using it or still considering?
}

\section{LI-RADS im Jahr 2020 - Nutzt du es schon oder überlegst du noch?}

Authors

Kristina Imeen Ringe ${ }^{1}$, Anne Gut ${ }^{2}$, Lars Grenacher ${ }^{3}$, Markus Juchems ${ }^{4}$, Guido Kukuk ${ }^{5}$, Johannes Wessling ${ }^{6}$, Andreas G. Schreyer ${ }^{7}$

Affiliations

1 Department of Diagnostic and Interventional Radiology, Hannover Medical School, Hannover, Germany

2 Department of Diagnostic and Interventional Radiology, Hospital Brandenburg, Germany

3 Imaging and Prevention Center, Conradia Radiology Munich, Germany

4 Diagnostic and Interventional Radiology, Hospital Konstanz, Germany

5 Department of Radiology, Hospital Graubünden, Chur, Switzerland

6 Department of Radiology, Clemenshospital GmbH Münster, Germany

7 Institute for Diagnostic and Interventional Radiology, Brandenburg Medical School Theodor Fontane, Brandenburg a. d. Havel, Germany

Key words

LI-RADS, liver, HCC, CT, ultrasound, MR-imaging

received 12.03.2020

accepted 21.06.2020

published online 20.07.2020

Bibliography

Fortschr Röntgenstr 2021; 193: 186-193

DOI 10.1055/a-1212-5915

ISSN 1438-9029

(c) 2020. Thieme. All rights reserved.

Georg Thieme Verlag KG, Rüdigerstraße 14,

70469 Stuttgart, Germany

Correspondence

Prof. Dr. med. Kristina Imeen Ringe

Diagnostische Radiologie, Medizinische Hochschule

Hannover, Carl-Neuberg Straße 1, 30625 Hannover, Germany

Tel.: ++49/511/5323424

Fax: ++49/511/53238 85

ringe.kristina@mh-hannover.de

\section{ABSTRACT}

Purpose The working group for gastrointestinal and abdominal imaging within the German Radiological Society performed a nationwide online survey in order to assess the current status regarding the awareness and application of LI-RADS, a classification for evaluation of liver lesions in patients at risk.

Materials and Methods Using the website www.deutscheskrankenhausverzeichnis.de a list of hospitals was generated meeting the criteria internal medicine, gastroenterology, general and visceral surgery and radiology $(n=391)$. Randomly, 102 department directors were contacted, and asked to name one consultant and one resident from their department in order to participate in the survey. 177 potential participants were invited to fill out an approximately 10 -minute online survey in the form of 17 questions regarding the awareness and application of LI-RADS. The results of the survey were analyzed by means of descriptive statistics.

Results 77 participants were registered, which corresponds to a response rate of $43.5 \% .47 \%$ of all participants were radiologists, $30 \%$ surgeons and $23 \%$ internal doctors/gastroenterologists, respectively, many with more than 13 years of professional experience (37.2\%). The majority of participants worked in a hospital with a focus $(37.2 \%)$ or a university hospital (29.1\%). Even though the majority of participants knows about or has heard of LI-RADS (73.2\%), only a minority uses the classification themselves ( $26 \%$ ) or within the context of tumor boards (19.2\%).

Conclusion The results of our survey demonstrate that LIRADS is relatively known in Germany, the application however quite sparse. This is in contrast to the general desire and endeavor for more standardized reporting in radiology.

\section{Key points}

- LI-RADS is not yet broadly implemented in clinical routine in Germany

- The sparse application is in contrast to the general desire for more standardized reporting in radiology

- Interdisciplinary education may support the propagation and use of the LI-RDAS classification

\section{Citation Format}

- Ringe KI, Gut A, Grenacher L et al. LI-RADS in the year 2020 - Are you already using it or still considering? Fortschr Röntgenstr 2021; 193: 186-193 


\section{ZUSAMMENFASSUNG}

Ziel In einer bundesweiten Online-Umfrage der Arbeitsgemeinschaft Gastrointestinal- und Abdominaldiagnostik der Deutschen Röntgengesellschaft erfolgte eine aktuelle Standorterhebung im Hinblick auf die Bekanntheit und Nutzung der LI-RADS (Liver Imaging Reporting and Data System)-Klassifikation bei der Beurteilung von Leberläsionen im Risikokollektiv.

Material und Methoden Über die Internetseite www.deut sches-krankenhausverzeichnis.de wurde eine Liste von Krankenhäusern generiert, welche die Kriterien Innere Medizin, Gastroenterologie, Allgemein- und Viszeralchirurgie und Radiologie erfüllten ( $n=391)$. Per Zufall wurden 102 Chefärzte der entsprechenden Abteilungen kontaktiert mit der Bitte, jeweils einen Fach- bzw. Oberarzt sowie einen Weiterbildungsassistenten zwecks Kontaktaufnahme und Teilnahme an der Umfrage zu benennen. Den insgesamt 177 eingeladenen Teilnehmern wurde mithilfe des SurveyMonkey ${ }^{\odot}$ Webtools eine Online-Umfrage mit 17 Fragen zum Thema LI-RADS und einer Bearbeitungszeit von etwa 10 Minuten zur Verfügung gestellt.
Die Ergebnisse der Umfrage wurden mittels deskriptiver Statistik ausgewertet.

Ergebnisse Insgesamt beantworteten 77 Teilnehmer den Fragebogen, entsprechend einer Rücklaufquote von 43,5\%. Bei den Teilnehmern handelte es sich zu $47 \%$ um Radiologen, zu $30 \%$ um Chirurgen und zu $23 \%$ um Internisten, der überwiegende Anteil mit mehr als 13 Jahren Berufserfahrung (37,2\%). Die Mehrheit der Teilnehmer arbeitete an einem Krankenhaus mit Schwerpunktversorgung (37,2\%) oder einem Universitätsklinikum (29,1\%). Auch wenn die Mehrheit der Teilnehmer (73,2\%) LI-RADS kennt bzw. schon einmal davon gehört hat, so wird LI-RADS lediglich von der Minderheit selbst (26\%) bzw. im Rahmen von Tumorkonferenzen (19,2\%) verwendet.

Schlussfolgerung Die Ergebnisse der Umfrage zeigen, dass die LI-RADS-Klassifikation zwar relativ bekannt ist, aber nur vereinzelt genutzt wird. Dies steht im Gegensatz zu dem allgemeinen Wunsch und Bestreben nach mehr standardisierter Befundung in der Radiologie.

\section{Introduction}

The LI-RADS (Liver Imaging Reporting and Data System) classification is a dynamic reporting system of the American College of Radiology (ACR), which was developed with the goal of standardizing terminology, technique and interpretation of findings in the context of liver imaging [1]. In addition to assessment of disease progression, use of the classification potentially also improves the interdisciplinary communication of findings. As a taxonomic system, the Reporting and Data Systems (RADS) of the ACR enable standardization of radiological findings and thus allow a structured classification of findings for a wide variety of clinical issues. Probably the best known and most established RAD system is the so-called BI-RADS (Breast Imaging Reporting and Data System), which is used in breast imaging [2].

The need for standardization and classification in liver imaging became clear in 2006 during a meeting of hepatologists and surgeons at the University of California San Diego, which drew attention to problems in the interpretation and especially interdisciplinary communication of CT and MRI findings [3]. The first LI-RADS version issued in 2011 resulted from a joint meeting of representatives of AASLD (American Association for the Study of Liver Disease) and OPTN (Organ Procurement and Transplantation Network) and has been continuously revised and developed since then (latest version 2018). Initially LI-RADS was developed only for CT and MRI (with extracellular contrast medium (CM)), but has since been expanded to include liver-specific contrast imaging in MRI and ultrasound including contrast-enhanced sonography [4].

LI-RADS is only used for patients with a high risk of hepatocellular carcinoma (HCC). Specifically, these are patients older than 18 years with known liver cirrhosis or chronic hepatitis B virus infection or patients with a known HCC. In principle, LI-RADS as- signs probabilities of HCC or benign masses to individual findings in the liver using a defined evaluation algorithm [1].

Since the 2017 version there has also been a German translation and accompanying manual for LI-RADS. The individual findings in the liver are called "observation" in the German translation. In principle, this term is used to describe every questionable finding in the liver, so that so-called pseudo lesions, such as those that can occur in the form of perfusion changes or artifacts, are also included [5]. It should be noted that the German translation of the LI-RADS version has not been reviewed and translated by the German-speaking radiological expert bodies and is not yet firmly anchored in a national guideline system in Germany or Europe.

LI-RADS assigns different categories to the observations that represent the probability that a lesion is HCC. As is typical for RAD systems, a five-stage system is used (LR-1 = definitely benign to $L R-5=$ definitely HCC). In addition, lesions can be described as LR-NC (non-categorizable) if the image quality of the underlying $C T$, MRI or ultrasound examination is insufficient. In addition, the LR-M category defines a liver mass that is highly likely to be malignant but highly likely not HCC, and the LR-TIV category defines a tumor infiltration into the vein $[1,6]$.

The classification of these LI-RADS categories is based on clearly defined "major features" or main criteria: arterial hyperenhancement, (non-peripheral) washout, increased contrast of a possibly existing capsule, the size of the observation and a possibly existing threshold growth ( $\geq 50 \%$ size growth in $\leq 6$ months). The first categorical classification of the individual observations is made based on these five main criteria. By using ancillary features, findings can then be downgraded by one category or upgraded to a maximum of LR-4 [1].

Originally it was planned to update the LI-RADS classification with a new version every 3 to 4 years [7]. However, this rhythm 
was already interrupted in 2018 in order to achieve compliance with the HCC guideline of the AASLD and the OPTN guideline on transplantation $[8,9]$. The integration of LI-RADS into US clinical guidelines was an important step towards the spread and acceptance of LI-RADS in the USA $[10,11]$.

In contrast, LI-RADS is not yet integrated into the current German and European guidelines. Although there is a clear trend towards structured reporting and standardization of terminology across all organs in the German-speaking world [12-14], the actual application still appears to be relatively limited. An additional problem for the acceptance of LI-RADS in the clinical routine is the still frequently changing versioning of LI-RADS and the resulting uncertainty among radiologists, but also among clinical partners in internal medicine, hepatology and surgery.

Due to the integration of the new LI-RADS version into the US guidelines and clinical evolution based on increased radiological literature on the topic, it is time to pay more attention to this system of structured reporting and terminology in Germany and Europe. This is also reflected in the significantly increasing number of publications on the topic. A PubMed search with the keyword search "LI-RADS" resulted in only one publication in 2012, whereas in 2020 (as of 03/03/2020) there were already 254 publications. There may be better acceptance in the future only if the system is adopted and increasingly propagated by the European and national professional societies.

For this reason, we decided to conduct a systematic survey of the status quo in an interdisciplinary environment via the German Radiological Society and the Working Group for Gastrointestinal and Abdominal Imaging of the DRG. The aim was to determine the degree of familiarity with the LI-RADS classification, the extent to which it is used in clinical routine and in the context of tumor boards, and in particular, the reasons for its lack of application. In this context, it should also be evaluated whether, from a clinical point of view, the use of such a classification is even considered necessary by the different disciplines.

\section{Materials and Methods}

After preparatory discussion among the experts involved, an online survey was conducted using the SurveyMonkey ${ }^{\odot}$ web tool (www.surveymonkey.com; [15]), which was made available in a professional expansion stage via the DRG. The survey consisted of 17 questions, of which 13 were multiple-choice questions and 2 each were decision (yes/no) and open questions. The survey was available between June 1 and July 31 2018, and consisted of three blocks of questions. The first block (5 questions) collected background information about the participants and their respective working environments. The second block (8 questions) covered awareness and use of the LI-RADS classification, and the third block (4 questions) addressed potential reasons for the lack of use of the LI-RADS classification and the general need for standardized reporting.

The target group of the survey were physicians from various disciplines involved in the diagnosis and treatment of patients with liver tumors, in particular gastroenterologists, hepatologists, general and visceral surgeons and radiologists. The Internet page www.deutsches-krankenhausverzeichnis.de was used to generate a list of hospitals meeting the criteria of internal medicine, gastroenterology, general and visceral surgery as well as radiology. From the resulting list of 391 hospitals, 102 clinics were randomly selected. In order to obtain a representative share from each German federal state, as many clinics were selected from each state proportionate to the respective share in the original list. The heads of the respective departments were contacted by email with the request to nominate a specialist or senior physician as well as a resident physician for the purpose of establishing contact and participation in the survey. The potential participants were invited over a period of 3 weeks up to a maximum of three times by telephone or e-mail to participate in the survey.

The SurveyMonkey ${ }^{\odot}$ tools were used to analyze the survey results. Further statistical analysis using descriptive methods was performed using Prism 7.0 (GraphPad Software Inc., San Diego, USA).

\section{Results}

After initial contact with the head physicians of 102 hospitals, we received the contact details of 177 potential contacts for the survey. Of these 177 invited physicians, 77 took part in the survey, representing a participation rate of $43.5 \%$. Since there was no mandatory answer to any of the questions, or if under certain circumstances they were not applicable, individual answers could be skipped.

\section{Section A: Background information and participant's working environment}

1. What is your specialty? (multiple answers possible)

2. How many years have you been clinically active (since completing your studies)?

3. What is your position in the hospital?

4. In which clinical environment are you involved?

5. How many beds are there in the hospital where you work?

Of the survey participants, $47 \%$ were radiologists, $(n=36), 30 \%$ surgeons $(n=23)$ and $23 \%$ internists or gastroenterologists $(n=18)$, mostly with at least 7 years of professional experience (67.5\%; Fig. 1). The environment consisted of a cross-section of resident physicians ( $37 \% ; n=28)$, specialists $(4 \% ; n=3)$, senior physicians (43\%; $n=33$ ) and attending physicians ( $17 \% ; n=13)$. Head physicians did not participate in this survey. Of the participants, $29.9 \%(n=23)$ worked at a university hospital $(n=23)$, $25.9 \%$ at a hospital providing maximum level 3 care $(n=20)$, $36.4 \%$ at a hospital providing priority level 2 care $(n=28)$, and $7.8 \%$ at a hospital providing standard level 1 care $(n=6)$. Regarding the number of beds at the respective facilities, hospitals with more than 800 beds predominated with $48 \%(n=37)$, followed by facilities with $400-800$ beds ( $39 \% ; n=30)$ and those with fewer than 400 beds $(13 \% ; n=19)$. 


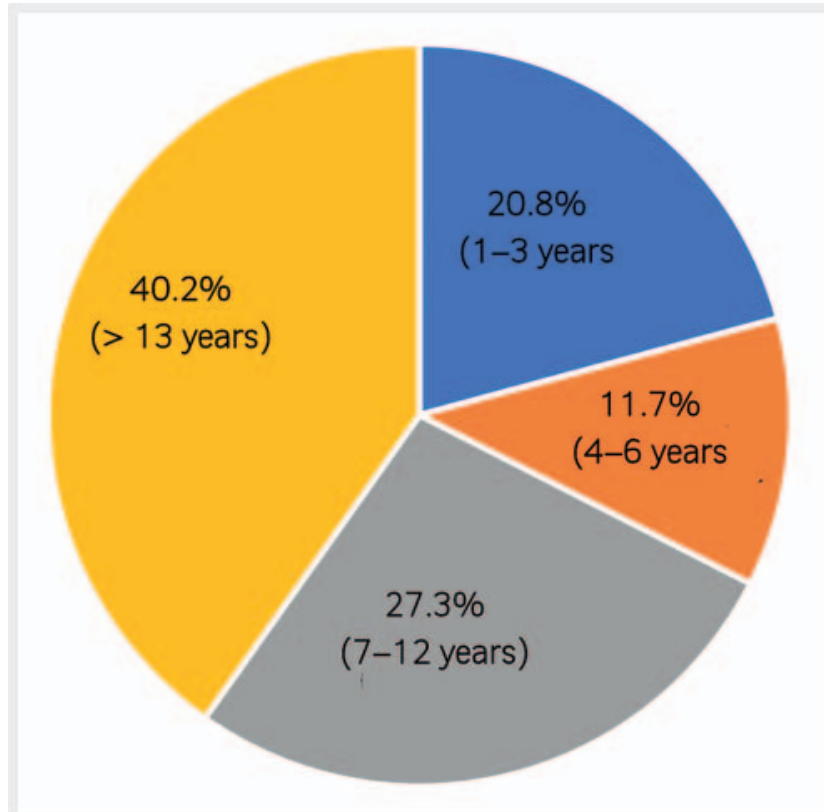

- Fig. 1 Responses to the question "For how long have you been working clinically?" Out of 77 participants, $40.2 \%$ have been working for more than 13 years, $20.8 \%$ were novices.

\section{Section B: Familiarity with and prior use of LI-RADS classification}

1. Are you familiar with the LI-RADS classification for the assessment of focal liver lesions in the risk cohort?

2. Do you believe that the application of the LI-RADS classification in clinical / everyday practice can standardize assessment criteria and reduce the scope of interpretation of findings?

3. How practicable do you find the LI-RADS classification for everyday clinical use?

4. Have you ever used the LI-RADS classification yourself?

5. Is the LI-RADS classification used in your clinic/department/ practice/tumor board?

6. Since when has the LI-RADS classification been used in your clinic/department/practice?

7. For which findings is the LI-RADS classification used in your working environment? (multiple answers possible)

8. How often is the LI-RADS classification used in your environment with respect to the relevant risk cohort?

Although the majority of respondents were familiar with the LI-RADS classification, it is striking that only $22.1 \%(n=17)$ indicated that they were well-informed about it. Most were radiologists (65\%), respondents who worked in a university environment (77\%) or in a senior medical or management position (88\%). The level of knowledge of LI-RADS as a function of the specialty surveyed is shown in Fig. 2 . The majority of the respondents (59.7\%) believed that the use of the LI-RADS classification in everyday clinical practice can standardize assessment criteria and reduce the scope for interpretation of findings accordingly. Only $7.8 \%$ held a contrary opinion, which is in line with the opinion that $61 \%$ of the survey participants consider the classification as it currently exists to be practicable ( $\bullet$ Fig. 3). This predominantly positive opinion clearly conflicts with the actual use of the classification in everyday clinical practice. Only $26 \%$ of respondents have used LI-RADS themselves, and only $19.5 \%$ indicated that LI-RADS is used in their clinic, department or tumor board. Of the 20 participants who have used the LI-RADS classification themselves, most were radiologists at universities ( $>$ Fig. 4$)$. When LI-RADS is used, then most often for MRI and CT imaging (37.5\%; $>$ Fig. 5), but overall the classification is only referred to in a small fraction of the findings of the applicable patient population. Of the survey participants $45.8 \%$ stated that LI-RADS is used in less than $50 \%$ of the corresponding findings ( $\triangleright$ Fig. 6 ).

\section{Section C: Reasons behind the lack of application; need for standardization}

1. Why is the LI-RADS classification not yet used in your environment? (multiple answers possible)

2. Would you like the LI-RADS classification to be applied (more) in your facility?

3. In general, would you desire more structured findings from radiology.

4. Comments/Notes

Among the 62 respondents who stated that LI-RADS is not used in their working environment, the main reasons for the lack of application were ignorance or inexperience with its use ( $n=19 ; 30.6 \%)$ and lack of practicability $(n=4 ; 6.5 \%)$. More than half of the participants $(n=32)$ stated that they did not know the reasons for the lack of application (51.6\%). Concerning the wish for an increasing application of the LI-RADS classification in the future, the majority of the respondents was circumspect; $57 \%$ answered accordingly with "maybe", however, $31.2 \%$ of the participants would like a corresponding application for all liver lesions ( $\triangleright$ Fig. 7). Interestingly, the majority of the survey participants wanted to see more structured findings from radiology $(52.1 \%)$ or structured findings for all oncological issues (42.9\%). The desire for more structured reporting in general was equally pronounced in all specialties; $97 \%$ of the participating radiologists (35/36) answered this question positively, as did $87 \%$ of surgeons (20/23) and $100 \%$ of internists or gastroenterologists (18/18). The desire for more structured reporting as a function of the clinical environment is shown in > Fig. 8.

\section{Discussion}

With a response rate of over $43 \%$, we were able to achieve a good and largely balanced survey result with a total of 77 participants in the various disciplines of radiology, internal medicine and surgery. To avoid a selection bias, all colleagues selected for the survey were personally contacted up to three times and reminded to stop the online survey. In this way we were able to reach a proportion of about $47 \%$ radiologists, $23 \%$ internists and $30 \%$ surgeons.

Overall, it can be summarized that the knowledge and application of LI-RADS varies greatly depending on the specialty, professional experience and clinical or academic environment. Participants from university clinics or large hospitals were most familiar 


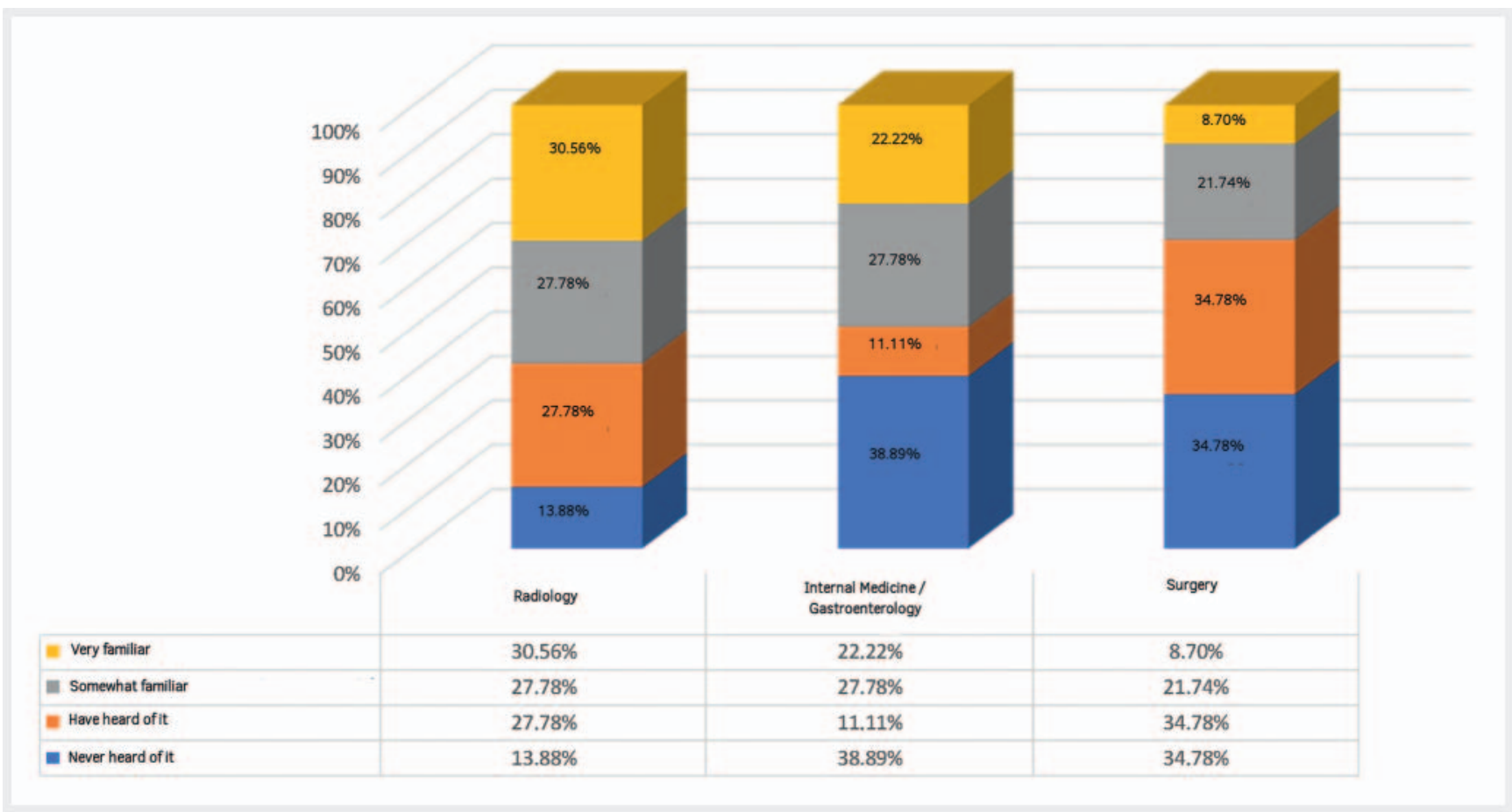

- Fig. 2 Responses to the question "Have you heard about the LI-RADS classification for evaluation of focal liver lesions in patients at risk?", as a function of clinical specialty.

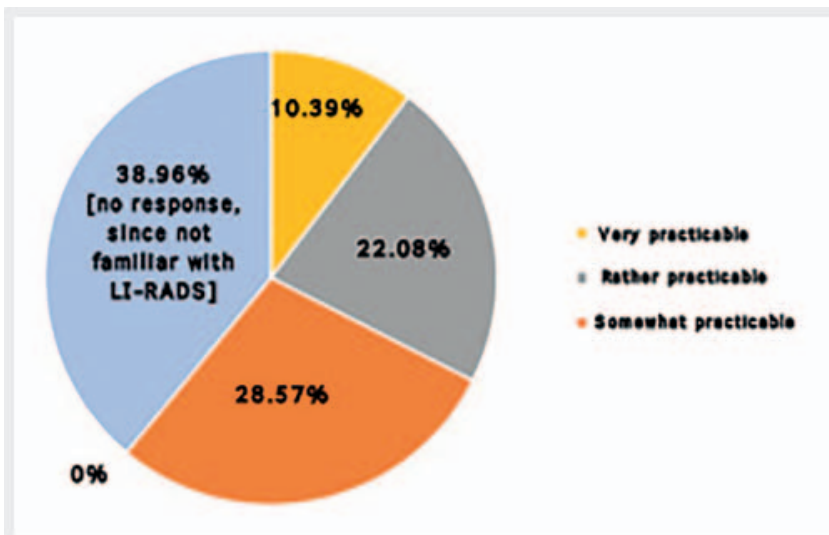

- Fig. 3 "How practical would you evaluate the LI-RADS classification?" Nearly $40 \%$ of participants ( $n=30$ ) were not able to answer this question due to lack of LI-RADS knowledge. On the other hand, more than $60 \%$ of the interviewed consider the classification as practicable.

with LI-RADS. With regard to specialty, participating radiologists had the highest knowledge rate of $86 \%$ ("at least have heard of LI-RADS"). Surprisingly, $39 \%$ of gastroenterologists stated they had never heard of LI-RADS, which was the case for $35 \%$ of surgeons and only $14 \%$ of radiologists. Although the LI-RADS classification and its creation by the ACR is a reporting system initiated by radiologists, it has an interdisciplinary approach and is designed to improve communication among different clinical disciplines. However, based on the results of our online survey, this hardly seems possible at the moment given the current level of knowledge and awareness of this classification.

In addition to the clinical environment, the educational or professional status of the participants is of crucial importance. The greatest unfamiliarity ("never heard of LI-RADS") was in the group of resident physicians with $32 \%$ (9 out of 28 participants), whereas this was the case for $18 \%$ ( 6 out of 22 participants) of senior physicians and $15 \%$ of attending physicians ( 2 out of 13 participants). Throughout the survey, however, it is noteworthy that the majority of respondents would in principle like to see structured reporting and structured terminology in radiology [14].

In the individual responses, the main reasons for the lack of application of the LI-RADS classification in clinical routine were the complexity of the system and associated difficulties. In particular, there was criticism that the "ancillary features" often contribute to additional lack of clarity. The complexity of the LI-RADS algorithm is indeed not unproblematic in everyday use, has already been criticized in the English literature and is a possible reason why it has not yet been integrated into additional guidelines [16, 17]. Regardless of the complexity of the system, many participants of the survey obviously do not even know why the LI-RADS classification is currently not used. This suggests that LI-RADSbased reporting may not yet have been discussed in the relevant institutes. An initial discussion in the individual departments and institutes could certainly help to spread awareness of the existence of the LI-RADS classification and ultimately its implementation. In addition, physicians who already use the LI-RADS system noticed that often not all necessary or required contrast medium phases were present during extramural examinations, so that an adequate LI-RADS evaluation in the context of tumor boards is of- 


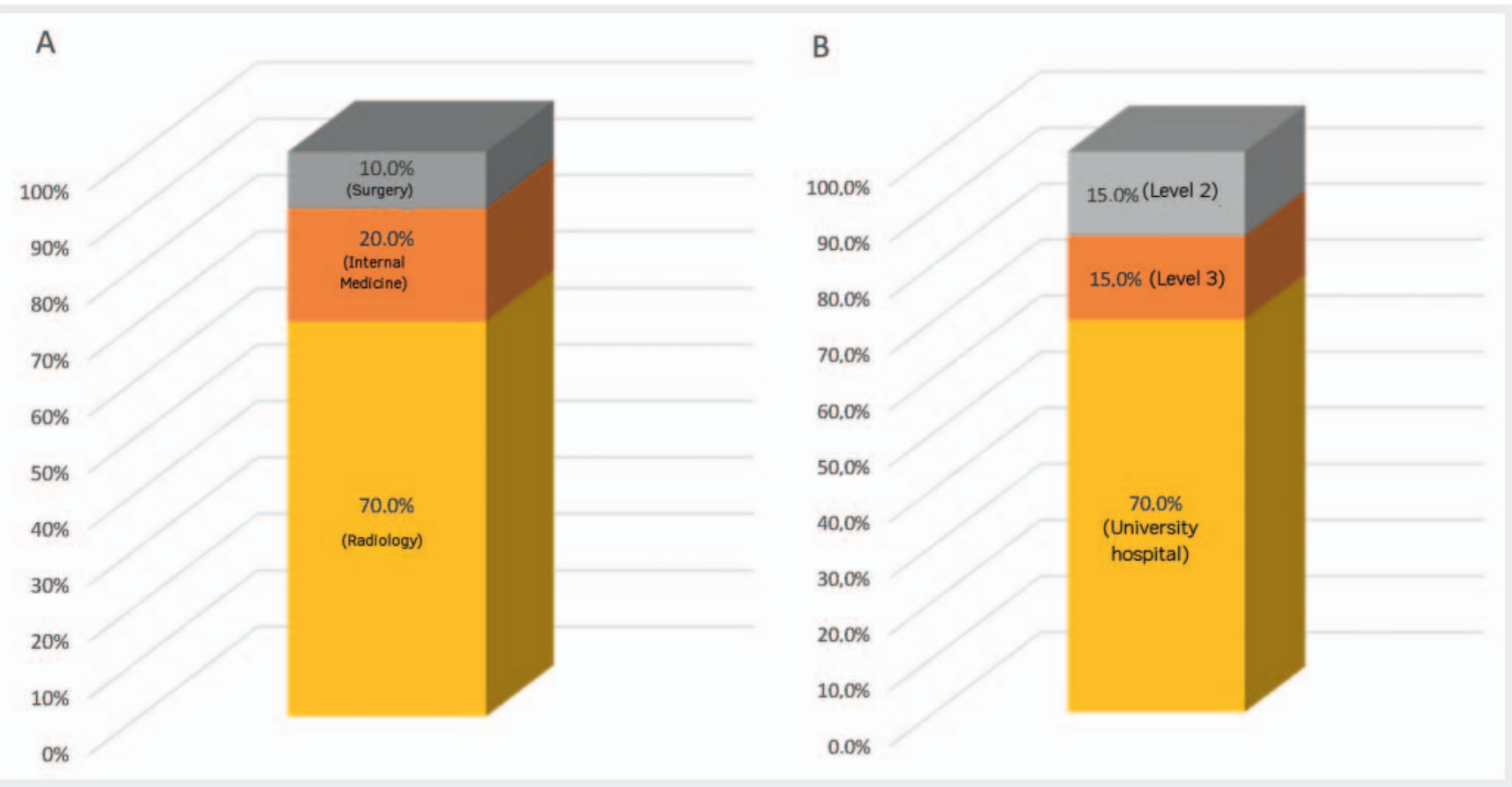

- Fig. 4 Reponses to the question "Have you yourself used the LI-RADS classification", as a function of clinical subspecialty (A) and clinical environment (B). Out of the 20 participants who have used the LI-RADS classification themselves, most were radiologists working at a university hospital.

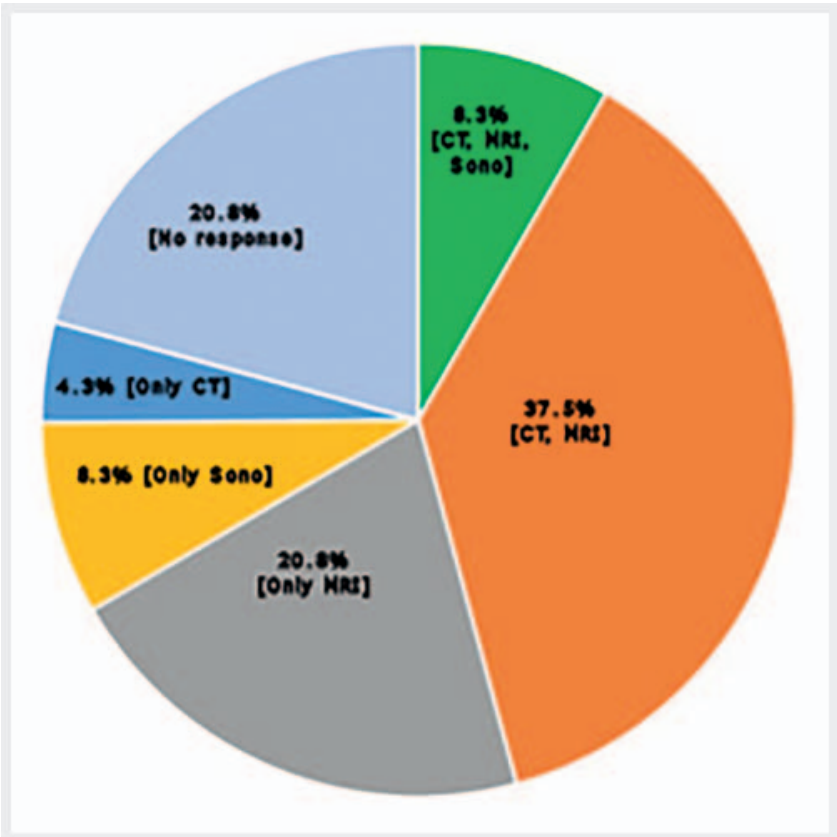

- Fig. 5 Responses to the question "For which modalities is the LIRADS classification used in your environment?" refer to 24 participants in whose environment LI-RADS is already in use. Strikingly, only 2 participants use LI-RADS for all modalities.

ten not possible [18]. This circumstance unfortunately confirms the lack of knowledge of the guidelines for the technical implementation of imaging of HCC [19] and a lack of in-depth discussion of LI-RADS assessments in the German Radiological Society.

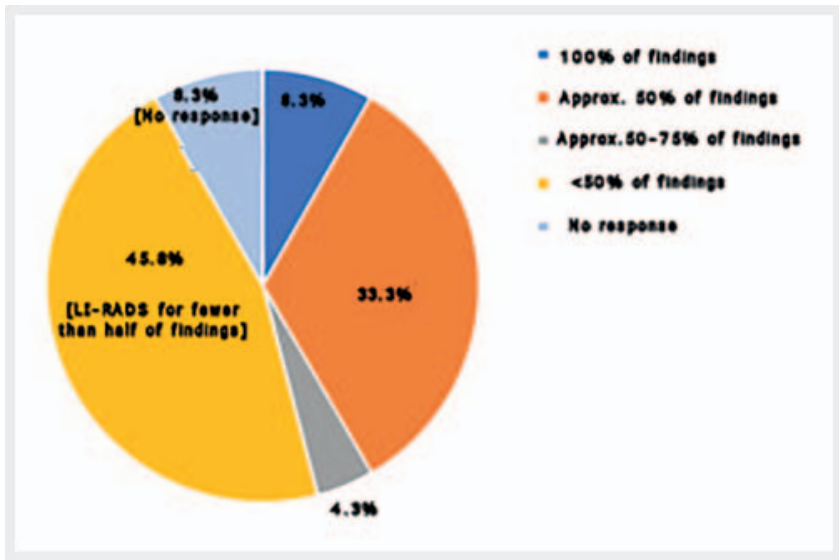

- Fig. 6 Responses to the question "How often is the LI-RADS classification applied in your environment for respective patients at risk?" refer to 24 participants in whose environment LI-RADS is already in use.

It also seems remarkable that there is only a low level of familiarity with the LI-RADS classification, especially among resident physicians. This may be partly due to the still low level of specialization during medical residency, but must be taken as an opportunity to implement more structured reporting in everyday clinical practice at this early stage, and to include RADS classifications in the curriculum of specialist training and actively make them available [20].

Another potential obstacle to the dissemination of LI-RADS may certainly be the language barrier of previous versions. Due to the recently available translations of the ACR in currently 9 lan- 
guages, a decisive contribution has been made to positively influence familiarity and application in German-speaking countries. Nevertheless, it must be critically noted that the translations of the ACR have generally not been officially performed under the

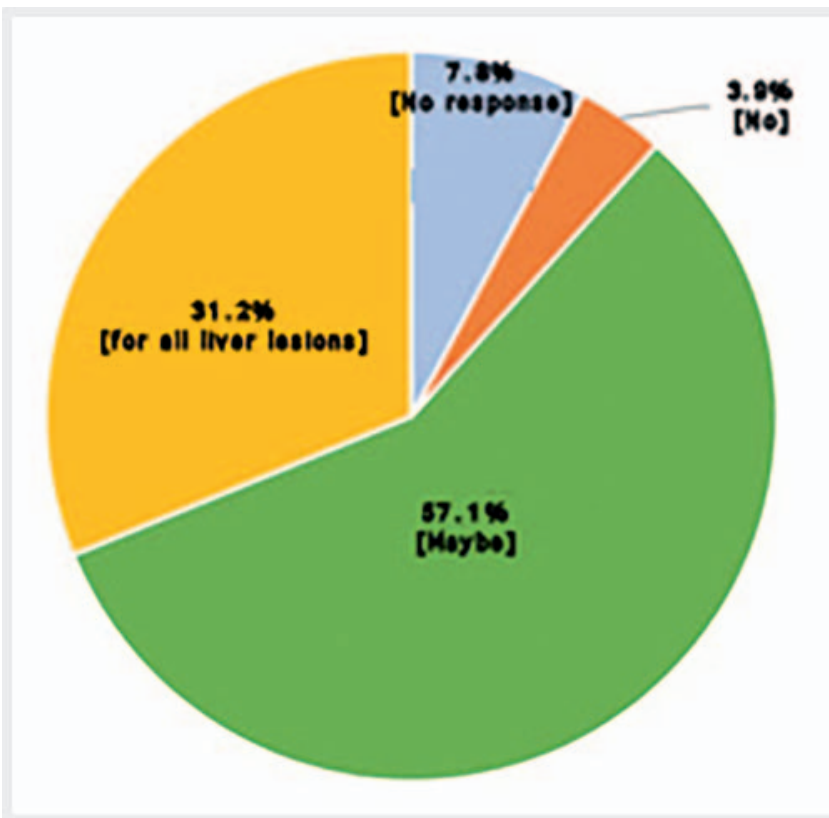

- Fig. 7 Responses to the question "Would you wish that LI-RADS is used (more) in your clinical environment?" A distinct minority (only $3.9 \%$ ) do not desire any LI-RADS application. auspices of the by the local radiological professional association and consequently sometimes contain somewhat ambiguous and unclear terms. A prime example is the word "observation", which in the strict sense of the Duden German dictionary has the implication of monitoring and is not ordinarily used in radiological language in Germany in the same English-language context [4, 21]. These relatively simple and misleading direct translations or even adoption of words into German will certainly not contribute to the acceptance of a classification, which should also be based on clear language that can be understood by all disciplines. Debatable are also formulations in terms of content, some of which are relatively vague, such as "multidisciplinary discussion of a targeted work-up (may include a biopsy)" as a recommendation for action in the case of LR-4 lesions.

In summary, it can be stated that, on the one hand, there is a great desire to increasingly use structured reporting in the German-speaking world (especially in oncological imaging), but that implementation has so far met with limited success. Joint training initiatives, for example through the DRG and its working groups, are certainly a first step towards this goal. At the same time, coordinated interdisciplinary training and dissemination of the LI-RADS classification must be implemented, especially since the recipients of radiological findings, namely hepatologists, gastroenterologists and surgeons, are still quite ignorant of this structured terminology. The third and probably decisive approach for broad application must be the integration of LI-RADS into local, in this case German or European guidelines on HCC. In Germany and in Europe it now depends on the new versions of the guidelines whether LI-RADS can be integrated into the European system. At the latest then, an increased knowledge of structured

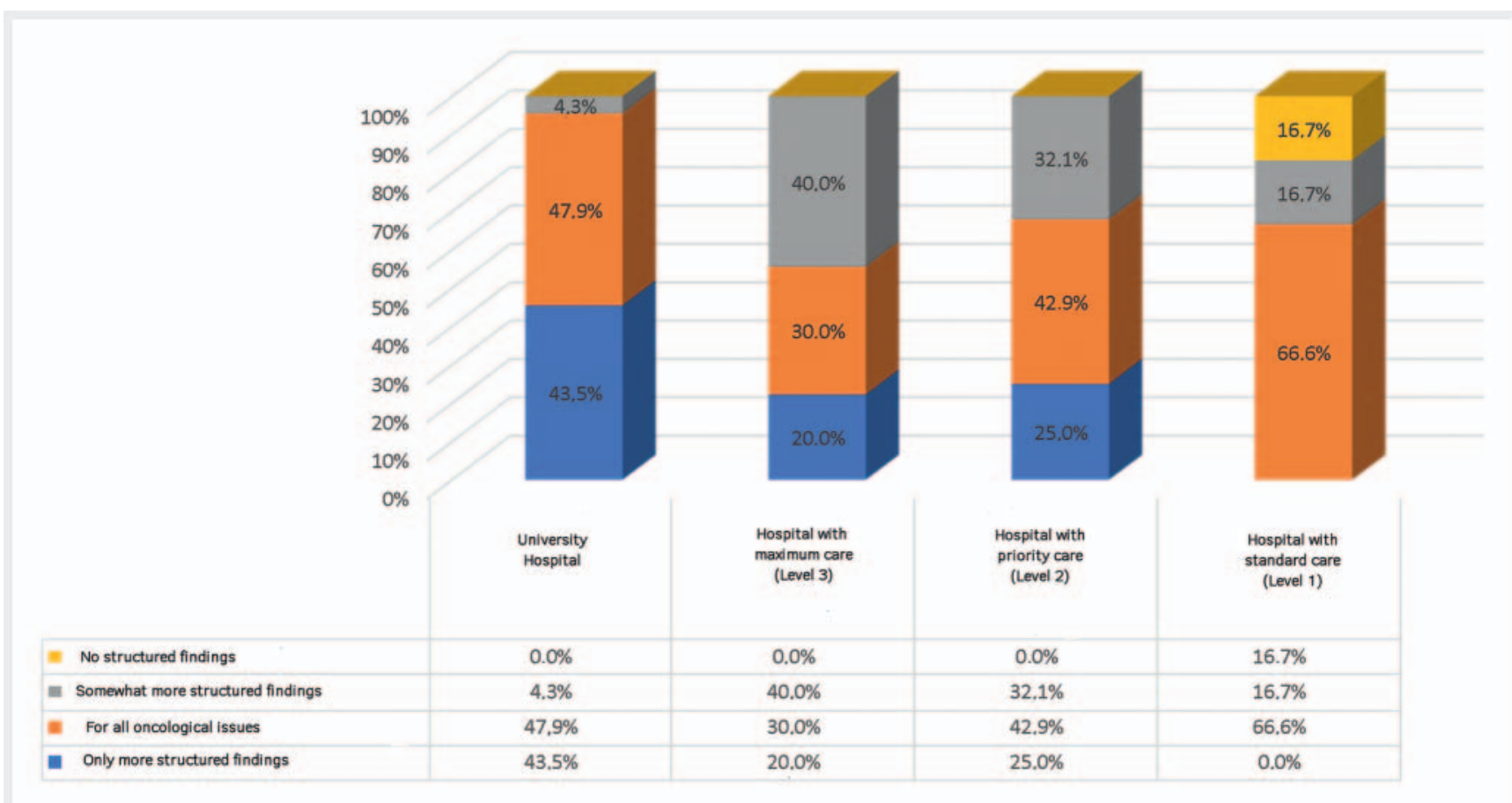

- Fig. 8 Responses to the question "Would you like to receive more structured reports from radiologists?" as a function of the clinical environment. Noticeably, a consistent desire for more structured reports can be identified. 
reporting according to LI-RADS is certainly possible and to be expected through the dissemination and application within the framework of the guidelines. An additional motivation for the increasing use of LI-RADS could also be its value as a potential prognostic tool, as recently shown in a study [22]. Finally, the lack or inadequate integration of structured reporting options into the currently installed radiological information systems (RIS) must also be critically noted [23]. In particular, computer-aided automation of data acquisition and documentation of an accepted standard, such as LI-RADS, is the decisive prerequisite for the seamless integration of such systems into the clinical routine.

\section{Conflict of Interest}

The authors declare that they have no conflict of interest.

\section{References}

[1] American College of Radiology Liver Imaging Reporting and Data System. https://www.acr.org/Clinical-Resources/Reporting-and-Data-Systems/LIRADS. Zugriff am 28.02.2020

[2] Mercado CL. BI-RADS update. Rad Clin N Am 2014; 52: 481-487

[3] Sirlin CB. The LI-RADS adventure-a personal statement. Abdom Rad 2018; 43: $1-2$

[4] Dietrich CF, Potthoff A, Helmberger T et al. Standardisierte Befundung und Dokumentation der Kontrastmittelsonografie der Leber (CEUS LI-RADS). Z Gastroenterol 2018; 56: 499-506

[5] Elsayes KM, Chernyak V, Morshid Al et al. Spectrum of Pitfalls, Pseudolesions, and Potential Misdiagnoses in Cirrhosis. Am J Roentgenol Am J Rroentgenol 2018; 211: 87-96

[6] Santillan C, Chernyak V, Sirlin C. LI-RADS categories: concepts, definitions, and criteria. Abdom Rad 2018; 43: 101-110

[7] Sirlin CB, Kielar AZ, Tang A et al. LI-RADS: a glimpse into the future. Abdom Rad 2018; 43: 231-236

[8] Marrero JA, Kulik LM, Sirlin CB et al. Diagnosis, Staging, and Management of Hepatocellular Carcinoma: 2018 Practice Guidance by the American Association for the Study of Liver Diseases. Hepatology 2018; 68: 723-750

[9] Kielar AZ, Chernyak V, Bashir MR et al. An update for LI-RADS: Version 2018. Why so soon after version 2017? J Magn Reson Imaging 2019; 50 : 1990-1991
[10] Goldberg-Stein S, Walter WR, Amis ES Jr et al. Implementing a Structured Reporting Initiative Using a Collaborative Multistep Approach. Curr Probl Diagn Radiol 2017; 46: 295-299

[11] Elsayes KM, Kielar AZ, Elmohr MM et al. White paper of the Society of Abdominal Radiology hepatocellular carcinoma diagnosis disease-focused panel on LI-RADS v2018 for CT and MRI. Abdom Rad 2018; 43: 2625-2642

[12] Pinto Dos Santos D, Hempel JM, Mildenberger P et al. Structured Reporting in Clinical Routine. Fortschr Röntgenstr 2019; 191: 33-39

[13] Pinto Dos Santos D, Arnhold G, Mildenberger P et al. Guidelines Regarding section sign 16 of the German Transplantation Act - Initial Experiences with Structured Reporting. Fortschr Röntgenstr 2017; 189: 11451151

[14] Herden U, Schoening W, Pratschke J et al. Accuracy of Pretransplant Imaging Diagnostic for Hepatocellular Carcinoma: A Retrospective German Multicenter Study. Can J Gastroenterol Hepatol 2019; 2019: 8747438

[15] Goldberg DTS, Maly T, Aldrich W. 2015 htpps://www.surveymokey.com

[16] Renzulli M, Clemente A, Brocchi S et al. LI-RADS: a great opportunity not to be missed. Eur J Gastroenterol Hepatol 2019; 31: 283-288

[17] European Association for the Study of the Liver. EASL Clinical Practice Guidelines: Management of hepatocellular carcinoma. J Hepatol 2018; 69: 182-236

[18] Kambadakone AR, Fung A, Gupta RT et al. LI-RADS technical requirements for $\mathrm{CT}, \mathrm{MRI}$, and contrast-enhanced ultrasound. Abdom Rad 2018; 43: 56-74

[19] Greten TF, Malek NP, Schmidt S et al. Diagnostik und Therapie die hepatozellulären Karzinoms. Z Gastroenterol 2013; 51: 1269-1326

[20] Siedlikowski ST, Kielar AZ, Ormsby EL et al. Implementation of LI-RADS into a radiological practice. Abdom Rad 2018; 43: 179-184

[21] Der Duden. www.duden.de. Zugriff am 28.08.2020

[22] Choi SH, Lee SS, Park SH et al. LI-RADS classification and prognosis of primary liver cancers at gadoxetic acid-enhanced MRI. Radiology 2019; 290: 388-397

[23] Flusberg M, Ganeles ], Ekinci T et al. Impact of a Structured Report Template on the Quality of CT and MRI Reports for Hepatocellular Carcinoma Diagnosis. J Am Coll Rad 2017; 14: 1206-1211 